Cultures \& Conflits

09-10 | printemps-été 1993

La violence politique dans les démocraties

europénnes occidentales

\title{
Cigaville : quand le maintien de l'ordre devient un métier d' expert
}

\section{Patrick Bruneteaux}

\section{(2) OpenEdition}

\section{Journals}

Édition électronique

URL : http://journals.openedition.org/conflits/223

DOI : $10.4000 /$ conflits.223

ISSN : $1777-5345$

Éditeur :

CCLS - Centre d'études sur les conflits lilberté et sécurité, L'Harmattan

Édition imprimée

Date de publication : 15 mai 1993

ISSN : 1157-996X

\section{Référence électronique}

Patrick Bruneteaux, «Cigaville : quand le maintien de l'ordre devient un métier d' expert », Cultures \&

Conflits [En ligne], 09-10 | printemps-été 1993, mis en ligne le 04 mars 2005, consulté le 30 mars 2021. URL : http://journals.openedition.org/conflits/223 ; DOI : https://doi.org/10.4000/conflits.223

Ce document a été généré automatiquement le 30 mars 2021.

Creative Commons License 


\title{
Cigaville : quand le maintien de l'ordre devient un métier d' expert
}

\author{
Patrick Bruneteaux
}

Il est possible de saisir les principes majeurs du maintien de l'ordre dans une "société démocratique" à partir, comme dit Mauss, d'un "fait social total" : On entend par là le fait que le centre de perfectionnement de la gendarmerie mobile, situé à Saint Astier, en Dordogne, est un condensé des principes essentiels du maintien de l'ordre, une sorte de revue de la panoplie "répressive" et des conditions d'application qui l'orientent. On peut ainsi faire l'économie d'une recension exhaustive des pratiques d'apprentissage puisque Saint Astier métonymise l'ensemble des répertoires de formation, actualisés et virtuels, de la gendarmerie mobile. Par ailleurs, Saint Astier donne à voir le monde de la gendarmerie mobile dans ses aspects les plus divers: métier, sociabilité de "cantonnement", loisirs, rivalités, cérémonies ${ }^{1}$. Le centre de perfectionnement de la gendarmerie mobile (CGPF), accueille tous les escadrons à intervalles réguliers (tous les deux ans généralement) pour un "stage" qui dure entre 10 et 15 jours. Pendant ce déplacement, c'est une sorte de révision générale des unités qui est effectuée par des cadres instructeurs permanents. Elle prend en compte l'ensemble des aspects jugés pertinents qui définissent à la fois "l'escadron opérationnel" et le gendarme mobile "capable de faire face à toutes les situations"2. Le symbole de cette instruction complémentaire est représenté par le "figuratif" du centre qui constitue une ville en miniature montée de toutes pièces pour les besoins de "l'évolution des formations". Saint Astier représente un concentré des rôles essentiels des forces de l'ordre, dont on retracera l'histoire "institutionnelle"3. Cette synthèse active du maintien de l'ordre révèle en fait une contradiction, puisque ce centre est voué autant à former des fonctionnaires spécialisés aptes à utiliser la force qu'à soumettre les gendarmes aux contraintes d'un maintien de l'ordre ouvert à la gestion du désordre toléré. Les agents qui incarnent le pouvoir de coercition étatique soulèvent la délicate question d'une socialisation en accord avec les nécessités objectives d'une institution qui offre au pouvoir politique la possibilité d'avoir le dernier mot. Il est donc essentiel que le gendarme ait les moyens matériels (physiques et techniques) et psychologiques ( tout ce que l'on mettra sous l'expression de "sens du devoir") de faire face et de s'imposer 
aux contestataires. "Force doit rester à la loi", telle est le précepte premier du corps. Par contre, le jeu politique démocratique a façonné un champ du conflit que les forces de l'ordre ont contribué à structurer ${ }^{4}$. Les gendarmes mobiles s'opposent donc aux militaires en ce qu'ils ne doivent pas détruire un ennemi, mais contrer "un citoyen momentanément égaré". L'instruction des personnels vise donc autant à façonner des individus qui ne doivent pas défaillir dans l'usage du "niveau" de force minimale à opposer aux manifestants, qu'à produire des dispositions de retenue, de "maîtrise de soi". La violence requise et la violence contenue représentent ainsi les deux faces de la police des foules.

\section{SAINT-ASTIER : I'UNIFICATION DU MAINTIEN DE L'ORDRE}

La préparation des militaires de la gendarmerie aux missions de défense civile commence véritablement après mai 68 . C'est en effet au lendemain de cette expérience limite du maintien de l'ordre que les problèmes de régulation du désordre contestataire se sont fait jour. Nul doute qu'après la "grande peur" des classes dirigeantes, le maintien de l'ordre soit devenu une priorité5. "Si l'un des premiers effets de mai 68 a été de reconstituer les dix escadrons dissous les années antérieures, l'effet majeur a été de créer une commission chargée de tirer les enseignements de ces soixante jours qui ont ébranlé la France"6. Et l'auteur de préciser que la nouvelle période est celle de la "revalorisation de la gendarmerie mobile par rapport à la gendarmerie départementale". Les "événements" rendent justifiée l'ouverture d'un centre spécial d'entraînement pour "moblots". La direction de la gendarmerie, décide de monter un Centre de Perfectionnement de la gendarmerie mobile qui s'ouvre en avril 1969 et fonctionne jusqu'en novembre 1970. Les équipes légères d'intervention sont expérimentées, sorte de commandos chargés d'attraper les "meneurs" les plus violents qui "font dégénérer les manifestations". Une sophistication des équipements est réalisée, notamment avec l'invention de casques spécifiques, de boucliers, de fourgonspompes, de grenades lacrymogènes aux effets variés. Le danger passé, la priorité est laissée à l'instruction des gendarmes auxiliaires, institution que l'augmentation des effectifs de la gendarmerie, suite aux luttes de mai-juin 1968, a justement rendue possible en préparant, dès le service militaire, le recrutement du personnel. En l'absence de centre unique, la "réflexion" se poursuit "dans les escadrons"7 , nombre de groupements disposant de terrains suffisamment grands pour prolonger les manoeuvres. Mais les difficultés nouvelles du maintien de l'ordre en milieu rural, illustrées notamment par les affrontements de Creys-Malville en juillet 1977 -c'est l'apparition du "maintien de l'ordre rural" 8 - conduisent les officiers à "réactiver" le Centre de Perfectionnement. Ce sont donc encore une fois des pratiques nouvelles de contestation, face auxquelles les forces de l'ordre semblent sur le point de n'être plus "opérationnelles", qui commandent la réouverture du "village de Cigaville". L'ouverture de ce centre était une innovation dans le corps. Elle posait d'autant plus de problèmes d'organisation qu'il devait s'agir non seulement d'un lieu de centralisation de toutes les expériences des unités mais aussi d'un espace d'expérimentation permanente. Cette nouveauté allait heurter les dispositions acquises : "A leur arrivée, les escadrons considéraient les stages comme un déplacement supplémentaire et montraient une certaine réticence à se comporter comme une école"9. Et, bien sûr, les 
premiers stages montrèrent que l'unification des pratiques autour des registres "modernes d'intervention" était une nécessité. Mais elle était loin d'être acquise, ce qui montrait bien la valeur du centre : "Aucune unité n'avait travaillé avec ce type d'engin (les Véhicules blindés à roue de la gendarmerie ou VBRG, fréquemment utilisés lors des luttes paysannes qui font intervenir à des buts "offensifs" les tracteurs, et plus rarement contre les transporteurs routiers). Beaucoup de gendarmes le voyaient pour la première fois. Compte tenu du peu d'entraînement des escadrons à travailler avec ces véhicules, il apparaît fort difficile de pouvoir définir actuellement une doctrine d'emploi. La période actuelle est une période de découverte"10. Les critiques pouvaient d'autant plus se faire jour que le centre avait justement pour fonction de traiter des "dysfonctionnements" qui ne pouvaient cesser immédiatement après les moments de crise qui l'avaient fait naître. Cette difficulté d'accepter le principe du "recyclage" reflétait un conflit entre l'inclination à l'autonomie des unités et la définition homogène d'une doctrine d'intervention plus ou moins imposée par les cadres de Saint Astier, tenus eux-mêmes de rendre compte de leur action au commandement des écoles situé à Maisons-Alfort. En fait, la mémorisation des épreuves de maintien de l'ordre rejouées à "Cigaville" allait d'autant mieux se banaliser que les exercices prenaient largement appui sur les expériences passées des escadrons et sur les demandes des officiers d'unités. Ainsi en est-il de la configuration même des lieux d'emploi. Le "figuratif" n'avait pas été conçu dans la première phase succédant à Mai 68. Autrement dit, il n'est pas sorti clé en main dans un projet mûri en commission; il résulte au contraire d'une réflexion empiriste tributaire d'une formulation des besoins dégagés de l'usage même du centre. "Saint Astier" est la somme des initiatives des cadres instructeurs, des suggestions des officiers de passage, des moyens budgétaires alloués et des imprévus contestataires. Entre 1968 et 1970, des travaux de déboisement avaient permis de disposer d'un "plateau d'évolution aménagé", autrement dit d'avenues artificielles élaborées avec des panneaux en ciment et un sol "urbain". La construction d'un "village" est pourtant sollicitée dans les rapports que les officiers d'unités communiquent à leur hiérarchie à la fin de leur stage. Certains proposent un "village de bois". Finalement, peu avant la réouverture du centre en 1977, une décision ministérielle entérine le projet de simulation d'un espace urbain: "Un figuratif d'agglomération rassemble un réseau de places, de symboles d'édifices et de rues suffisamment dense pour permettre toutes les manoeuvres concevables en zone urbaine. Une ébauche d'usine sert en outre au déroulement des différentes opérations que les forces de l'ordre sont appelées à mener dans ce genre d'établissement"11. Par la suite, des aménagements ont été opérés sans remettre en cause la modalisation ainsi définie $^{12}$ : les voitures (qui sont incendiées lors des assauts des plastrons ou qui sont heurtées dans un "nettoyage de barricade" par le blindé-lame) ont été récupérées dans des "casses" ou offertes par des gendarmes ou des particuliers ; des commerçants ont été satisfaits de faire de la publicité gratuite en installant des enseignes; des officiers ont proposé des outils supplémentaires, tels qu'une illustration sonore du bruit symptomatique des manifestations, au moyen d'un mégaphone. Enfin, la définition des "stages" - qui se sont resserrés de 20 jours à la fin des années 1970 à 10 depuis le milieu des années 80 - a correspondu à la volonté de conserver les traces du passé et de s'en servir comme mémoire, retransmise à toutes les unités. Le cycle d'instruction est ponctué par des conférences sur les "tactiques de l'adversaire" éprouvées par certains escadrons et analysées au profit des unités en stage, par des films sur les grands moments de contestation ${ }^{13}$. C'est ce souci de conservation des rigueurs du passé 
lointain comme du passé proche d'ailleurs, qui a pour effet d'instituer un décalage entre les scènes jouées et la réalité des conflits sociaux à un moment donné. Mais surtout, la mémoire des affrontements se conserve dans la série des "exercices tactiques" que les unités rejouent en actes, avec la complicité des "plastrons"14 ("dispersion foule calme puis houleuse", "évacuation d'un local de nuit", "interdiction d'accès à un édifice public", "charge et réduction de barricades", "action de décrochage", etc.). Autrement dit, les forces de l'ordre évoluent dans des situations de confrontation qui commencent avec la gestion de manifestations pacifiques et culminent avec des "rétablissements de l'ordre"; ils autorisent le recours à des armements plus dangereux (grenades à souffle dites "OF", action des VBRG, droit de marquage physique plus étendu). Variations graduelles des répertoires qui permettent l'adaptation générale des répertoires des forces de l'ordre aux répertoires des contestataires. On peut ajouter que les instructeurs du centre collectent aussi l'ensemble des comptes-rendus d'intervention que chaque commandant de compagnie est obligé de rédiger à la suite d'un déplacement effectué dans le cadre de ce qu'on peut appeler la police des foules. Il faudrait pouvoir retrouver systématiquement les récits que les officiers échangent lors de leurs séjours et qui font partie intégrante du processus de circulation des informations. Il semble que ce centre d'entraînement en soit venu à constituer une mémoire propre, un monument en soi, digne d'intérêt et de célébration. Le centre de perfectionnement tend à construire une sorte d'idéal de professionnalisme de la gendarmerie mobile, et finalement une image valorisante. Au tout début, les officiers de la gendarmerie recherchaient la discrétion la plus totale. Aujourd'hui, il n'est pas rare de voir des journalistes se déplacer à "Cigaville" pour prendre quelques photos ${ }^{15}$. Le centre est devenu aussi un lieu de démonstration des méthodes de la gendarmerie, en faveur d'autres corps de police ${ }^{16}$.

\section{LA VIOLENCE REQUISE}

5 Cette expression veut suggérer le fait que l'intériorisation des techniques de corps (au sens individuel et collectif) n'est jamais acquise, en ce qui concerne les pratiques de maintien de l'ordre. L'existence de Saint Astier montre que la formation initiale dispensée dans les écoles de base des sous-officiers et des officiers ne suffit pas à préparer les gendarmes à leur future mission dans un escadron. C'est ainsi que l'on fait $l^{1}$ hypothèse d'une instruction permanente ${ }^{17}$ qui permettra, à chaque individu, selon sa position dans l'unité, de conserver le "niveau" de force suffisant pour faire face aux exigences du maintien de l'ordre. Deux facteurs majeurs expliquent ces "déficiences structurelles". La première réside dans le décalage entre la multiplicité des répertoires formels d'intervention et les aléas des types d'action. Un escadron peut ne pas avoir rencontré tel scénario -par exemple une évacuation d'usine - depuis plusieurs mois voire plusieurs années. Le second facteur aggrave le premier. En moyenne, depuis les années 1970 jusqu'à aujourd'hui, les unités consacrent entre 25 à $30 \%$ (selon les années) de leur activité totale aux charges de maintien de l'ordre ${ }^{18}$. En conséquence, l'exigence d'unification des pratiques astreint chaque unité à maîtriser l'usage de l'ensemble d'un répertoire qu'elle n'utilise jamais totalement en déplacement.

6 Une force qui se refait une forme La priorité donnée à la "récupération" des forces individuelles (éducation physique, sport...) et collectives (connaissances techniques et 
manoeuvres pratiques) s'illustre tout d'abord dans l'organisation même de la vie quotidienne dans le centre. Depuis sa création, tout a été fait pour en faire le lieu idéal $\mathrm{d}$ '"une remise en condition". Après mai 68 , la première génération de recyclés a connu une transposition à Cigaville des règles internes aux unités. Chaque escadron devait se prendre en charge ${ }^{19}$. Dans sa nouvelle formule, inaugurée en 1977, le centre prend la forme d'une organisation de "stages" sur le modèle de l'entreprise. Tous les aspects de garde, d'entretien sont pris en charge par la structure d'accueil et les personnels ne seront plus distraits par les nécessités de la "logistique". C'est un engagement total des gendarmes dans les exercices qui devient possible. En fait, ce lieu se distingue aussi de la vie ordinaire au cantonnement en ce qu'il ne fait pas alterner les temps de "M.O." et le reste ${ }^{20}$. Pendant une quinzaine de jours, c'est enfin la sociabilité propre à la gendarmerie mobile qui s'exprime, en marge des profanes (la famille, les "civils"). Ce milieu offre à l'inverse un "concentré de rôles" lié au maintien de l'ordre et qui se tient dans un emploi du temps sans failles. Tous les rapports $(\mathrm{N}=27)$ notent que "au plan psychologique, le séjour a raffermi la cohésion des unités et a permis à chacun de se mieux connaître"21. Situation unique, tout le groupement est présent, du gendarme au lieutenant-colonel. Les trois escadrons qui le constituent se retrouvent avec l'étatmajor qui les coiffe ; et tous évoluent ensemble sur les circuits. Moment rituel où tout le groupe se rapproche physiquement, spatialement, relationnellement. La force des unités, c'est d'abord leur unité, une hiérarchie pleinement reconnue, une communauté qui s'affirme aussi par delà la différenciation des postes et des grades à travers des moments fusionnels: "L'une des meilleures images du séjour est ce tour d'honneur de l'escadron victorieux, saxophone en tête, passant devant l'unité battue, qui applaudissait généreusement les camarades porteurs de la coupe (...) L'une des meilleure images du séjour est l'arrivée de la marche chronométrée du $7 \mathrm{Km}$ où gradés, anciens et jeunes gendarmes, trouvaient encore les ressources pour entonner "La Madelon" à l'entrée de la dernière ligne droite. L'autre bonne image est celle des personnels à la piscine où du jeune gendarme au commandant de groupe, ils sautaient à la queue leu leu depuis le plongeoir de trois mètres" ${ }^{22}$. De façon plus générale, la dureté des épreuves physiques et des manoeuvres, le nombre élevé d'exercices de confrontation, servent à induire un processus de solidarité collective dans la résistance commune aux violences endurées : "Plus on prend de coups et plus on est solidaires"; "Quand ça commence à chauffer, c'est là qu'il y a le plus d'entraide. On se soutient plus et on tient mieux" ${ }^{\prime 23}$. Dans les premiers temps de son incorporation, la nouvelle recrue appréhende son nouveau métier, notamment lorsqu'il s'agit de rester en position de "barrage fixe fermé", sans bouger ni reculer alors que les manifestants lancent des projectives à propos desquels chacun sait qu'ils feront des blessés ${ }^{24}$. Le rôle du "chef" est alors de "rassurer", "de montrer l'exemple", de "repérer les fragiles" : "Quand il y a des blessés ou que l'attente devient interminable, tout le monde se tourne vers le chef. c'est lui doit avoir la présence d'esprit de ne pas se couper de ses hommes. Il doit montrer qu'il n'a pas peur. il faut qu'il montre son autorité" 25 . La recherche des conditions optimales de performance qui place les gendarmes déplacés à St Astier dans un univers relativement inversé où de nombreux agréments leurs sont octroyés (prise en charge administrative, installations de détente, relâche de la rigueur hiérarchique), reflète l'ambiguïté d'une institution de contrôle ${ }^{26}$ qui, sans prendre la fonction explicite d'une inspection, correspond en pratique à une évaluation de la valeur "opérationnelle" de chaque escadron. La création de St Astier, au lendemain de crises du maintien de l'ordre, signifiait à chaque fois une volonté de reprise en main des 
formations, une stratégie d'homogénéisation vers le haut. L'unification des usages possibles des escadrons ${ }^{27}$ imposait la création d'un contrôle systématisé du label: "gendarmerie mobile", qui se concrétise dans une "doctrine d'emploi" de plus en plus fouillée ${ }^{28}$. Elle fût d'ailleurs, pour partie, impulsée politiquement ${ }^{29}$.

\section{Les pratiques d'insensibilisation à la violence}

Disposer d'une violence mobilisable selon un niveau d'intensité donné ne signifie pas que les personnels sont formés à donner des coups de matraques. Etrangement, cela ne s'apprend pas. La force de frappe proprement dite est une opération individuelle qui n'est pas enseignée ou révisée dans les situations de simulations du centre. Le travail de correction vise uniquement les formations (différents types de barrage), les évolutions des unités ${ }^{30}$, et principalement la résistance aux ripostes des contestataires ainsi que les manoeuvres de dégagement ou de dispersion. Les épreuves destinées à tester la solidité des unités, à la consolider voire à l'améliorer prennent, bien sûr, appui sur une fiabilité globale des escadrons, et cela d'autant plus qu'ils sont déjà passés à plusieurs reprises au centre d'instruction. En cela fait total, la formation dispensée laisse voir tout un ensemble d'acquis, jamais pris en compte directement à Cigaville comme base d'exercice, mais sans lesquels la révision des dispositifs d'intervention n'aurait aucune chance d'être efficace. Il s'agit de la sociabilité de résistance collective propre à l'escadron. Ces cent hommes occupent chacun une place déterminée ${ }^{31}$, et côtoient toujours les mêmes partenaires lors de leurs déplacements. L'interconnaissance des "assiégés" a pour conséquence le fait que chacun anticipe les réactions de ses voisins, et sait sur qui compter. De même, chaque escadron arrive à Saint Astier avec un personnel déjà plus ou moins formé, armé de la considération qu'il s'attribue, à la suite de ses "épopées" ${ }^{32}$. On pourrait même se demander si, avec le déclin relatif des luttes violentes dans les années 1980, Saint Astier ne devient pas la figure substitutive des références marquantes qui font tenir les gendarmes dans les moments difficiles. Le capital d'expérience accumulé dans les conflits est à la fois utilisé et testé à Cigaville. Certaines techniques, utilisées de façon routinière dans le centre, visent à rappeler cette présence du groupe à lui-même, notamment les actions symboliques de synchronisation; ce qui se joue à travers l'abaissement prompt et simultané des casques ou encore dans la frappe des bâtons de défense sur la partie intérieure des boucliers, lorsque les escadrons se trouvent en position de barrage, ce n'est pas seulement d'impressionner les manifestants par une telle unicité des réflexes. On peut y voir aussi un rappel "vibrant", comme une métonymisation de l'existence du groupe en chacun ; bruit régulier et rythmé dont l'effet de puissance, auquel chacun participe, le traverse et le convainc de la présence active de tous les membres de l"'unité" et le rassure ainsi sur sa force. Les passages dans le village obéissent certes à un but explicite : faire que les personnels puissent s'approprier en situation les différents répertoires d'action. Mais en fait, derrière la recherche de la maitrise de ces outils, c'est surtout la "maîtrise de soi" qui est recherchée. Le fonctionnaire ne doit pas fuir, comme l'image sur un mode humoristique un officier chargé du recrutement : "Vous imaginez un gendarme effrayé devant des manifestants agressifs et qui, dans un mouvement de panique, grimperait à un arbre ?" 33 . Etre à la hauteur des propres registres de la gendarmerie mobile, voilà le défi de St. Astier. L'apprentissage du maintien de l'ordre commence avec l'obligation de 
supporter d'être incommodé par les fumées lacrymogènes. Bien que dotées de masques, il est fréquent que les unités en respire ${ }^{34}$. Il n'est pas rare de voir certains gendarmes évoluer au milieu des gaz. L'ethos impose simplement de pleurer et de cracher tout en restant à son poste. L'indifférence, feinte ou réelle, avec laquelle les gendarmesplastrons accueillent les jets de grenades lacrymogènes et surtout les grenades offensives $^{35}$ montre que la valeur pratique des forces de l'ordre commence par leur capacité à supporter les effets des techniques utilisées contre les contestataires. Il faut ainsi qu'ils soient en quelque sorte à même de devenir insensibles à leur propre violence, avant de l'administrer aux autres. Selon les unités et la mentalité de l'officier qui dirige à un moment donné le centre, l'insensibilité peut constituer non seulement une défense mais un véritable principe d'honneur. L'entraînement devient alors une série de défis à travers lesquels les unités jaugent leur vaillance :"J'en suis à mon 5ème passage et il faut dire que les engagements physiques semblent moins durs actuellement qu'ils ne l'étaient au début des années $1980^{36}$. "Il y a un fait qui mérite d'être souligné. Il y avait avant le Lt colonel L.G. qui apportait aux exercices son "animation personnelle" et, de ce fait, les affrontements étaient plus acharnés. Cela ne veut pas dire que la pédagogie y gagnait" ${ }^{37}$. L'encouragement que l'encadrement apporte à ces actions d'endurcissement ${ }^{38}$ semble avoir été la règle pendant quelques années, sans que le niveau de violence atteint ne plaise à tout le monde ${ }^{39}$. Le "sangfroid" ou le "calme" des troupes de manoeuvre ont pour fonction de faciliter l'adaptation à la violence adverse, de demeurer vigilant en vue de pouvoir s'adapter à tous les scénarios. Lorsqu'il s'agit d'un exercice de "maintien de l'ordre face à une foule pacifique", il ne faut pas que ce soient les forces de l'ordre qui introduisent un désordre supérieur à celui qui est nécessaire pour maintenir le désordre adverse dans les limites acceptées ${ }^{40}$. Inversement, lorsque les manifestants sont "virulents", les réactions des gendarmes sont testées au moyen d'indicateurs limites. Leur calme est évalué par le lancement de cocktails molotov sur les premiers rangs. En cas de prise du feu sur la tenue ignifugée, le gendarme doit demeurer impassible et attendre que la "sécurité" vienne à son secours. La résistance à la fatigue s'éprouve entre autre dans le rapport au temps, avec des cadences de travail inaccoutumées, des exercices de nuit ; la complexité des scénarios vise à évaluer l'"adaptabilité manoeuvrière" des escadrons, par le biais de changements de posture ${ }^{41}$, ou la création d'imprévus de dernière minute. De façon plus générale, Cigaville est une recherche de simulation réaliste qui vise à placer tous les gendarmes dans des affrontements relativement durs de telle sorte qu'ils ne soient pas pris au dépourvu si l'occasion se présentait. En effet, nombre de gendarmes mobiles ou mêmes d'escadrons n'ont pas connu de situations vraiment "tendues" : Les exercices de Cigaville permettent "d'appliquer les techniques dans des situations réalistes, parfois difficiles, en donnant ainsi l'occasion d'acquérir les réflexes nécessaires en étant confrontés, bien sûr toutes proportions gardées, à la violence verbale et gestuelle de la foule, aux bruits, aux fumées, à la mise à feu de barricades, au lancement de bouteilles incendiaires, à toutes sortes de projectiles. Le souci de la direction d'exercice est de calquer au maximum les exercices sur des opérations qui ont eu effectivement lieu. Et vous savez, pour beaucoup de jeunes gendarmes, tout cela constitue une découverte" ${ }^{142}$. 


\section{LA VIOLENCE CONTENUE}

9 Alors que la violence requise vise à inculquer les dispositions convenables d'un gendarme mobile apte, dès lors, à se conduire avec les attitudes attendues de "fermeté" et "d'énergie" nécessaires ${ }^{43}$, la notion de violence contenue se définit comme la modalité du répertoire ayant pour but de contraindre l'emploi de cette force ainsi créée. La contradiction principale de toute "formation générale" au maintien de l'ordre vient de l'obligation de faire "des hommes aptes à l'effort"44, sans que la force ainsi créée se déploie librement, comme à la guerre où la destruction de "l'ennemi" est recherchée. A terme, "le plus dur ce n'est pas d'envoyer les hommes mais de les retenir"45. A fortiori, la force de frappe du groupe est accentuée, en situation, par les réactions d'emportement qui peuvent $s^{\prime} y$ ajouter. L'instruction permanente vise à inculquer aux "assiégés" un potentiel de résistance hors du commun, de mesure dans l'action, d'inhibition dans la propension à la vengeance. Plus particulièrement ici, on va voir comment les forces de l'ordre gèrent leur propre violence en jouant surtout sur sa représentation auprès des manifestants; et comment, en cas d'insuccès relatif, elles intègrent les données de "l'énervement" en vue, dans la mesure du possible, de les anticiper.

10 La force contre la violence Bien avant les tournées à Saint Astier, les gendarmes reçoivent une interprétation "légaliste" et "pacificatrice" du maintien de l'ordre ${ }^{46}$. Tout au long de leur formation initiale, puis par la suite dans les unités ${ }^{47}$, les gendarmes apprennent à accepter "le sacrifice" d'un rôle ingrat qui les amène à "un haut sentiment du devoir", autrement dit au don de soi au groupe (Mauss). Et c'est ce "dévouement mutuel", ce "loyalisme", ce "culte du devoir"48 qui en fait une force de travail étatique disponible pour des interventions pénibles, retraduites en pratique, par la suite, selon la formule : "on sert de fusible" ; "on est là pour encaisser" ${ }^{49}$. Les qualités de disponibilité sont d'autant plus intériorisées qu'elles s'ancrent dans une perception de la réalité qui définissent leurs adversaires comme irrationnels. La théorie de la foule, inculquée tant aux cadres qu'aux exécutants, leur apprend à ne plus prendre au premier degré les agressions dont ils sont l'objet. Les injures perdent leur charge provocatrice. Ce n'est plus telle personne singulière qui s'en prend à eux personnellement, mais un individu parmi d'autres et qui aurait tout aussi bien pu se défouler sur un collègue. La réduction du contestataire à une entité abstraite dépersonnalise la relation de confrontation. Elle favorise la mise en oeuvre des normes techniques d'auto-contrainte en exerçant sur le garde une influence modératrice engendrée par le recul des tendances à la vengeance. Comment en vouloir à un "citoyen momentanément égaré" ? ${ }^{50}$. Car cette disposition au contrôle de soi est animée par la croyance en la supériorité psychologique du garde mobile sur le manifestant. "Lui" "connaît" l'état mental de "suggestion" du contestataire pris dans une foule ${ }^{51}$. Sur le plan des représentations, les gardes mobiles assument en quelque sorte le rôle d'une médecine des foules. A Cigaville, les assauts des plastrons sont accompagnés d'injures retournées dans le sens de la dérision: "Moblot, salaud, le peuple aura ta peau". Ils crient, chantent, injurient, et cette représentation théâtrale vide de sens le contenu des slogans ou des récriminations proférées à leur encontre.

11 Le contrôle du répertoire Le maintien de l'ordre ne repose pas exclusivement sur la dose d'abnégation du gendarme mobile. Les dispositifs et les moyens matériels qui définissent le "style" de ce corps s'articulent aux dispositions d'auto-contrainte et leur 
permettent de "tenir". Le maintien de l'ordre commence par une action de symbolisation de la dureté, stratégie première dans l'évitement des affrontements. L'investissement codifié de la rue se réduit d'abord à l'adoption d'un barrage de corps qui représente une forme défensive statique composée d'hommes concentrés et protégés. Dès lors, offrir l'impression d'une troupe indéfectible est une façon de compenser l'efficacité toute relative des barrages. Un comportement inflexible refroidit souvent les ardeurs : "Il ne faut jamais parler aux manifestants. Le fait de s'adresser à eux, c'est supposer qu'on prend fait et cause pour eux" ${ }^{152}$. L'effet d'intimidation appelle toute une préparation, à commencer par l'invisibilité de la mise en place du barrage ${ }^{53}$. L'uniformité des gestes commence dès la descente des cars : "Il faut que tout le monde descende d'un pas décidé en sachant où aller !" ${ }^{54}$. Face à la foule, "l'attitude militaire irréprochable" s'impose à tous les figurants. Elle proscrit tout "relâchement", exige un "silence total", implique de "rester calme et impassible", ce qui signale la disposition d'"être toujours prêt à intervenir". Elle commande aussi d'avoir "une attitude qui en impose". En effet, "une attitude résolue peut éviter d'intervenir en impressionnant les manifestants". Plus précisément, devant des manifestants, il faut toujours "se montrer ferme, énergique; ne manifester aucune nervosité, aucun signe d'impatience, aucune marque de fatigue; rester indifférent aux cris, menaces, injures et même aux applaudissements"55. A un stade supérieur, lorsqu'"il n'est plus possible de tenir", qu'"il faut se dégager", "souffler un peu", "prendre de l'air"56, les forces de l'ordre chargent. $C^{\prime}$ 'est l'aspect le plus impressionnant ${ }^{57}$. Mais il ne faut pas oublier que, outre la rareté quantitative des charges par rapport au nombre d'interventions, le profane ne voit pas que la plupart des courses ont pour fonction non pas d'atteindre le manifestant mais d'éviter, au contraire, un contact possible qui tournerait au corps à corps ${ }^{58}$. La charge proprement dite, avec usage des "bâtons de défense", représente soit un dernier recours après avoir tenté de tenir le plus longtemps possible, soit une stratégie explicite de dispersion "par la force" sur ordre du préfet. Si la majeure partie des interventions est constituée effectivement par des stratégies défensives, le maintien de l'ordre est constitué majoritairement par des temps d'attente. Or, la formation vise à inculquer cette capacité de résistance (au temps, à la chaleur ou au froid, à l'énervement). Mais celle-ci a ses limites. Comme tout individu prisonnier d'une situation à risque, les gendarmes en arrivent à vouloir "en découdre", "pour en finir". Le cumul de la fatigue (attente dans les cars et dans les dispositifs statiques, faim, injures, blessures) introduit un passage insensible de l'assurance à la crispation : "Il y a une tension qui augmente de plus en plus", "ça devient une sorte de règlement de compte", "on est des hommes et on répond"59. Pour contrer les risques de défoulement que le maintien de l'ordre "passif" implique, les officiers ont progressivement mis au point des techniques spécifiques qui ont pour fonction de maintenir les forces dans l'ordre, dans une discipline contenue : par exemple, "il faut économiser les hommes et si vous ne le faites pas, c'est là que vous avez les mauvaises réactions"60. La formation des officiers au CPGM consiste à ce propos à leur apprendre à "alléger leur dispositif dès que la situation le permet", à "mettre le nombre précis de pelotons nécessaires"61. La notion de "réserve" constitue ainsi une notion clé du maintien de l'ordre. Il faut toujours que le personnel puisse se reposer après avoir "camphré". Le repos est perçu comme la condition d'équilibre d'agents qui doivent demeurer autant que possible "maitres de soi", "sans esprit de vengeance", "sans réactions individuelles"62. Ainsi, lorsque les unités doivent intervenir dans un contexte "extrêmement hostile" et que l'unité a déjà subi des "pertes", le commandement tente dans la mesure du possible de 
relever l'escadron afin que "la charge ne soit pas une décharge"63. A Cigaville, l'opération est répétée dans le cadre d'un exercice type. La nouvelle équipe pousse à pas chassés ceux qui quittent le contact et deviennent des "réserves"64. La technique du décrochage représente un répertoire destiné à éviter un corps à corps qui induirait des conditions propices à des réactions armées de "légitime défense". "Opération pénible, mais on est pas là pour gagner"65. Enfin, la conservation d'une action collective "déterminée mais sans violences inutiles"66, impose une capacité d'appréciation différenciée de la violence des adversaires. Les interventions sélectives sont apparues après mai 68 lorsque furent créées les équipes légères d'intervention (ELI). Sorte de commandos de l'escadron, elles sert à la saisie des "meneurs" venus "pour faire dégénérer la manifestation". Dans ce cas, "on va pas s'en prendre à toute une foule alors que seul un individu isolé lance des pierres. Souvent, si un type isolé frappe, il n'est pas possible d'intervenir, les autres vont payer pour lui"67. Une doctrine spécifique ${ }^{68}$ détaille les modalités d'arrestation de ceux qui, selon les officiers, remettraient en cause le bon fonctionnement de la manifestation et de la modalité du répertoire qui l'aménage aussi. Ainsi, loin de se réduire à de l'acharnement ou à de "la violence colérique" 69 , ni même à une pure réaction mécanique de répression sur ordre du pouvoir politique, le maintien de l'ordre en France est devenue une affaire de professionnels. La complexité des manoeuvres et le coût temporel, financier, de préparation des escadrons à la gestion des luttes sociales et politiques, sont là pour témoigner de l'existence d'une répression (au sens d'Elias) de la répression.

\section{NOTES}

1. On profitera donc de l'évocation des expressions de sociabilité observées à Saint Astier pour se pencher plus globalement sur ce thème de la "cohésion".

2. Education physique, sport, parcours, conférences sur "les matériels", "la foule et le manifestant", les "techniques d'intervention etc., films sur des manifestations (mai 68, Creys-Malville...), "exercices tactiques" sur le terrain obéissant à une progression qui fait défiler l'ensemble du répertoire d'action contestataire.

3. On ne retient ici de l'analyse institutionnelle que le méthode sélective des moments créateurs ou "instituant", en mettant de côté les concepts d'"implication" ou de "transversalité". En fait, prendre le parti de sélectionner les pratiques fondatrices revient, dans un autre langage, à répertorier "les aspects pertinents" qui représentent les transcriptions pratiques de "stratégies" : L'analyse institutionnelle organise une lecture inédite de événement ou du phénomène étudié qui privilégie non sa description mais sa signification. L'accent est mis sur la dynamique sociale, l'action de l'instituant, les temps d'inflexion et de rupture, les phases de développement et de dépérissement", Antoine Savoye, « Du passé, faisons l'analyse, le traitement de l'histoire », in R. Hesse et A. Savoye, Perspectives de l'analyse institutionnelle, Klincksieck, 1988, p 156.)

4. La relégation des forces armées du terrain de la lutte sociale et la création en 1921 des pelotons mobiles de gendarmerie, puis en 1927 de la garde républicaine mobile, enfin en 1944 de la gendarmerie mobile et des compagnies républicaines de sécurité, 
ont été la marque exemplaire d'une rupture dans la conduite des opérations de police collective. La constitution d'un répertoire d'action spécifique à des forces d'intervention dites de "seconde catégorie" signale la genèse d'une violence autocontrainte de l'Etat de type démocratique, travaillée par les contraintes d'un "maintien de l'ordre" dans des conflits de plus en plus ouverts.

5. Voir notamment James Sarazin, Le Système Marcellin, Calmann-Lévy, 1974, p 69.

6. Conférence du Lieutenant-colonel de gendarmerie R...à Nice, à des officiers des CRS en 1990, document remis par l'auteur à Saint-Astier.

7. Op. cit.

8. Les années 1970 voient apparaître des mobilisations écologistes puis paysannes qui se singularisent des manifestations classiques par le choix de cibles (respectivement les centrales nucléaires et les camions sur les routes) qui font évoluer les contestataires et les forces de l'ordre sur des terrains aux configurations non prévisibles (champs, axes routiers, voies ferrées...etc.). La complexité des "manoeuvres" imposées par la diversité des lieux et des stratégies de lutte (éparpillement, commandos, bluff), a fait l'objet de recherches d'une "doctrine d'emploi", qui se sont concrétisées dans "l'instruction sur le maintien de l'ordre en milieu rural du 20 mars 1985".

9. Rapport du chef d'escadron directeur du CPGM, 7 novembre 1977, considérations initiales de fonctionnement du CPGM, Archives contemporaines de la gendarmerie, centre administratif de Leblanc, dossier E 451.

10. Ibid.

11. Décision ministérielle $\mathrm{n}^{\circ} 55080$ du 26 novembre 1976, in "Dossier de stage", document consulté à Saint-Astier.

12. Le terme de modalisation, qui constitue une transformation d'une partie du réel social ou naturel, est employé par Goffman pour désigner les relations d'interaction qui dérivent d'un "cadre primaire" ; ces concepts sont abordés le premier chapitre de son livre Les Cadres de l'expérience, Ed. de Minuit, 1992. Cigaville représente une reproduction d'un quartier réel redessiné en fonction des besoins du lieu.

13. Mai 1968, Creys-Malville, Plogoff, et plus récemment l'événement de Bourg d'Iré à l'occasion duquel un Lt. colonel, s'était rendu sur place pour tourner un reportage exploitable pédagogiquement pour les stagiaires de St. Astier.

14. Un plastron est une modalisation possible des manifestants. En effet les gendarmes qui se mettent en représentation sous la figure du "meneur" savent jusqu'où ils peuvent aller dans les agressions qu'ils commettent à l'égard de leurs collègues. Cette astuce pratique est empruntée à l'univers militaire qui l'emploie depuis le début du siècle. Par ailleurs, les CRS employaient ce registre pédagogique dès les années 50 ; A N, archives des CRS, cote 890672 art.3, Procès-verbal de la réunion des commandants du 5ème groupement, 11 juin 1955.

15. Lors de mon déplacement, un reportage a été effectué. Ces reportages concernent la presse écrite. En particulier, Sud-Ouest du 29 décembre 1987, article de Dominique Delaage ; Le Monde, du 6 juin 1990, article de Erich Inciyan. Ces papiers montrent que le journaliste est attiré par une originalité qu'il transforme en "spectacle", en événement "cocasse", notamment avec des titres et sous-titres révélateurs : "Quand les moblots jouent aux casseurs", "le bal est ouvert", "la valse des barricades". Par ailleurs, un numéro spécial de la revue de la gendarmerie paru en 1988 fut le premier du genre à consacrer deux articles à Saint Astier. Enfin, n'est-il pas significatif qu'un chercheur ait pu être autorisé à évoluer pendant plusieurs jours en toute liberté sur le camp ? Les officiers disaient alors : "Nous n'avons rien à cacher". 
16. Pour les élèves-commissaires qui consacrent depuis peu quelques jours de leur formation à la connaissance de la gendarmerie mobile. Plus globalement, de nombreuses délégations de gendarmeries et de polices étrangères ont été reçues. 17. Qui au pire se fait dans l'unité, comme c'est le cas pour certaines CRS qui, ne disposant pas d'un camp militaire, évoluent dans leur compagnie en se réservant une journée par semaine et une semaine par an pour les répétitions jugées nécessaires. 18. Archives des CRS, documents statistiques, cotes 890672 art 1 et 890466 art 7 , années 1973 à 1978 ; Direction de la gendarmerie, documents statistiques du bureau opération pour la période 1990-1992.

19. "Chaque escadron contribue au fonctionnement du CPGM en mettant à sa disposition 8 gendarmes pendant toute la durée du stage. Lorsqu'une unité est de semaine, ce sont 10 gendarmes qui sont soustraits journellement de l'instruction auxquels il faut ajouter le personnel indispensable à la bonne marche de l'escadron" Rapport du capitaine commandant l'escadron 4/2, 6 février 1970, Archives de Leblanc, E 541.

20. Les actions de renfort de la gendarmerie départementale, d'instruction militaire, de surveillance des frontières...

21. Rapport du chef d'escadron commandant le groupe 2/2, 18 février 1970, Leblanc, cote E 451 .

22. Ibid.

23. Discussions informelles avec des gradés sur les lieux d'exercice, Saint-Astier. Je me souviens notamment d'un gradé qui devint en fait un véritable informateur, livrant les aspects les plus secrets de la vie d'un escadron, c'est à dire les "adaptations secondaires" parfois illégales (comme manger de la tortue en Nouvelle-Calédonie ou tirer des lapins aux abords des pistes d'Orly) qui dessinent véritablement les configurations singulières de solidarité et de temps forts qui distinguent les escadrons. 24. Les escadrons "jeunes" se distinguent des plus anciens par le fait que, dans les premiers affrontements rencontrés, le barrage d'homme se contracte insensiblement, les corps se resserrent et se touchent. Les murs attenant aux trottoirs ne sont dès lors plus tenus. Dans l'incertitude des coups reçus individuellement, le groupe fait corps et "accorde" ainsi sa force organique à chacun (remarque faite par un capitaine, direction de la gendarmerie, septembre 1992).

25. Entretien avec un capitaine commandant un escadron, Saint-Astier.

26. De contrôle interne de la valeur respective des escadrons.

27. vécue par les plus anciens commandants comme l'arrivée d'une "sophistication qui devient inapplicable", entretien avec un chef d'escadron "affecté" dans la garde républicaine, et proche de la retraite, Paris, Mars 1989.

28. L'instruction 7001 de 1975 , qui est le terme de toutes les innovations qui ont suivies Mai 68 régit sur près de 150 pages les principes du maintien de l'ordre dans la gendarmerie mobile. Texte novateur qui n'a d'équivalent que dans l'instruction du 1er Août 1930, revu en 1945. Il aura fallu toutefois le réveil des "événements" pour que la direction de la gendarmerie refonde l'ensemble de dispositions abandonnées largement à des textes anciens, autrement dit, à "l'initiative" pratique des commandants d'unité. 29. La décision ministérielle du 26 décembre 1976, qui définit les missions du CPGM, reconnaît explicitement cette fonction de "revivification" - et par là de recherche de rétention de la violence - en posant comme premier principe du "programme général d'instruction" le "recyclage dans le commandement et l'exécution des différentes manoeuvres classiques du maintien de l'ordre". La consolidation des unités, telle 
qu'elle découlait de l'instruction 7001, imposait par ailleurs "un perfectionnement réalisé à partir d'informations réelles les plus récentes de maintien ou de rétablissement de l'ordre, sur les mentalités et les méthodes des adversaires" et, troisième principe, "un entraînement sportif soutenu de nature à donner aux personnels un allant et surtout une condition physique indispensable pour supporter les efforts intenses et les lourdes fatigues pouvant résulter des activités de maintien de l'ordre", Dossier de stage.

30. Sans pouvoir s'étendre sur la question des répertoires d'action des forces de l'ordre, on en mentionnera quelques une pour mémoire : "vague de refoulement", "bond offensif", "charge", "évacuation d'une usine", "destruction de barricade", "saisie de manifestants", "décrochage", "remplacement d'une unité mise en réserve".

31. Une division du travail répressif très rigoureuse sépare les fonctions des boucliers, face aux manifestants, et qui protègent l'unité, les grenadiers, qui lancent les grenades lacrymogènes et les éléments mobiles qui font les charges.

32. Les affrontements les plus durs contribuent à produire la valeur effective de l'unité, conservés dans les mémoires mais aussi consacrés par les lettres de félicitations individuelles et collectives adressées par le préfet et les officiers supérieurs. Les grandes dates entendues le plus souvent et vécues par les personnes interrogées sont : "Creys-Malville" ; "Plogoff" ; "Denain" ; "Golfech" ; "Chooz" ; "Les étudiants -1983" ; "Bourg d'Iré" ; "les étudiants -1986". Le sentiment de sa force propre s'enracine aussi à partir des classements qui distinguent les unités dans les championnats sportifs internes.

33. Entretien avec le Lieutenant-colonel chargé de l'animation d'une équipe $d$ 'officiers et de psychologue travaillant à la conception des procédures de recrutement du personnel de la gendarmerie, direction de la gendarmerie, Août 1989.

34. Du fait des vents contraires, d'une charge qui conduit l'escadron dans le nuage destiné aux manifestants (ici le plastron), du temps perdu à mettre le masque (sans parler de l'étanchéité toute relative des lunettes de protection) et surtout, à Cigaville, $\mathrm{du}$ fait que les plastrons ne se gênent pas pour ramasser les grenades et pour les renvoyer sur des unités qui, en position immobile de barrage, ne doivent pas bouger d'un pouce.

35. Dont l'effet de souffle a terrassé pourtant plus d'un lors des exercices, mêmes si les mesures de sécurité imposent aux lanceurs de tirer dans des endroits vides prévus à cet effet.

36. Entretien avec un chef d'escadron, Saint-Astier.

37. Entretien avec un colonel au colloque international de la gendarmerie, juin 1991.

38. Qui semblent aussi provenir de mécanismes extérieurs à St Astier. En effet, il semblerait que la diminution de la proportion des interventions "actives" dans les manifestations par rapport aux déplacements M.O., chiffrée à $3 \%$ par le commissaire Berlioz lors de la journée d'étude sur le maintien de l'ordre du 27 septembre 1991 (organisée par le Centre de Recherche Politique de la Sorbonne) déclenche des réactions de frustration. Cigaville aurait alors une "fonction parallèle" de défoulement. 39. "L'entraînement n'est plus ce qu'il était. Il y a quelques années, les gars préféraient descendre dans le rue face aux bretons les plus redoutables plutôt que d'aller se cogner à Cigaville. On avait quinze blessés par exercice de synthèse, des bras cassés, de tout. Ca allait trop loin. désormais c'est plus calme". Cité par Dominique Delaage, Sud-Ouest du 29 décembre 1987. 
40. Le colonel qui contrôlait l'opération émit les critiques suivantes, lors du débriefing qui réunissait des élèves-officiers : "C'est une situation calme, il faut seulement un peloton d'alerte prêt à gicler; il faut mettre les véhicules plus loin que la mairie. Vous risquez des états-d'âme des autorités civiles : la vie continue !! il ne faut pas faire débarquer les escadrons ; si vous n'avez pas pour ordre d'interdire la circulation, vous laissez passer. C'est pareil pour les civils qui vont acheter en ville", Saint-Astier.

41. En faisant alterner par exemple le maintien de l'ordre urbain et le maintien de l'ordre rural, ou le maintien de l'ordre et le rétablissement de l'ordre

42. Entretien avec le Lieutenant-colonel commandant les stages à Saint-Astier.

43. Cette terminologie est celle employée par les préfets lorsqu'ils attendent de la force publique qu'elle disperse des manifestations "interdites", présupposant avec évidence que les forces de l'ordre vont déployer une force suffisante pour faire fuir les contestataires récalcitrants.

44. Formation des formateurs, stage pédagogique, support de cours, réalisé par les instructeurs de l'école des sous-officiers de Chaumont, document consulté à MaisonsAlfort, commandement des écoles.

45. Evoqué dans un entretien avec et par Dominique Monjardet, qui rapporte les propos de gradés et d'officiers de CRS. Cette phrase se retrouve aussi dans la plupart des commentaires des officiers de la gendarmerie.

46. Les manuels de "formation morale et civique" dissèquent longuement les institutions politiques et le rôle de la gendarmerie à propos de son rôle de défense des libertés publiques, les conditions précises qui légitiment une arrestation, et donne une version détaillée du comportement acceptable du gendarme. par exemple, le manuel de maintien de l'ordre de 1969 pour gradés et gendarmes commentent : "le but de la formation individuelle : elle a un but moral qui doit développer le sens de la discipline, le sens du devoir, l'esprit de solidarité, le culte de la légalité, le respect de la dignité humaine. Elle a un but physique qui procure...la stabilité émotionnelle indispensable au cours des opérations de maintien de l'ordre. Elle a un but technique qui tend à l'acquisition...des réflexes nécessaires pour qu'en toute circonstance la mission de maintien de l'ordre s'accomplisse avec le minimum de dommages pour les parties en présence". Centre de documentation de la direction de la gendarmerie.

47. Où l'instruction continue, notamment par le biais de conférences "morales" du commandant.

48. Ces expressions proviennent du manuel du gendarme au maintien de l'ordre, 1969, op cit.

49. Entretiens avec d'anciens gendarmes promus officiers, $40 / 45$ ans. Saint-Astier. Le Manuel de formation morale et civique des gendarmes précise : "Tout militaire de la gendarmerie doit se pénétrer de l'idée que l'exercice de droits relativement étendus comporte en contrepartie des devoirs et des astreintes".

50. Conférence prononcée par un cadre du CGPM à une promotion d'élèves-officiers à Saint-Astier, sur le thème "La foule et les manifestants", Juin 1989, cette expression reprend celle qui est officiellement inscrite dans l'instruction 7001, op cit.

51. Comme le psychanalyste qui refuse de prendre au pied de la lettre les agressions qui dérivent du transfert. Le degré de normalité du manifestant est parfois l'objet de boutades inventées plus ou moins librement : "Pour connaître la mentalité d'un manifestant pris dans une foule, vous prenez le plus con et vous la divisez par le nombre de manifestants". 
52. Entretien avec un Lieutenant-colonel instructeur à l'école des officiers de Melun, Saint Astier.

53. Véritable contrôle de la région postérieure, ou, autrement dit, des coulisses, qui en dit long sur la recherche du contrôle des impressions produites : "Le débarquement s'effectue hors de la vue et du contact des manifestants", manuel de formation morale, op cit.

54. Critique de la descente des cars, par un Lieutenant-colonel lors du débriefing après l'exercice, Saint Astier.

55. Instruction 7001, op cit.

56. Notes prises au cours des critiques d'exercices faites par les instructeurs aux élèvesofficiers. Saint Astier.

57. "Je n'ai jamais vu charger des barricades, mais quand on a vu une fois une charge de police à Paris, c'est quelque chose qu'on n'oublie pas. Ils chargent au pas de course. Ils semblent invincibles" in Chroniques de mai 68, Mavis Gallant, Deuxtemps, 1988, p 18.

58. Les "bonds offensifs", réalisés sur une dizaine de mètre ont pour objectif de repousser des manifestants qui "viennent au contact". Les grenades lacrymogènes sont très souvent utilisées, avant de disperser, dans le but d'éviter tout rapprochement entre les contestataires et les forces de l'ordre ; ces contacts étant jugés propices à des actions incontrôlées de part et d'autre. Par ailleurs, les "vagues de refoulement" instrumentalisent une dispersion sans volonté d'atteindre l'adversaire.

59. Entretien avec des gardiens devenus officiers, op cit. L'un d'eux, ancien gradé, racontait : "Il y a un moment où c'est plus possible de supporter. On devient attaquant, surtout les types qui sont au contact. Alors, au moment de la charge on a ses têtes. Bien sûr le chef gueule. Il dira "du calme" et se base sur ces gradés pour repérer les gars qui s'énervent. Si la charge a lieu et "si le gradé veut y aller, le capitaine peut rien faire. c'est le gradé qui mène l'action. Alors les gars se libèrent",

60. Lt-colonel instructeur à l'école des officiers de Melun.

61. Notes prises au cours de critiques d'exercice d'élèves-officiers, Saint Astier.

62. A cet égard, les gendarmes mobiles ont un point d'honneur, celui de ne pas faire selon eux de "course à l'échalote" ; expression qui désigne les poursuites individuelles et que stigmatise l'instruction de référence du corps; Ils se plaisent à dire que ce sont des corps de police tels que les compagnies d'intervention de Paris qui donnent les scènes de tabassages collectifs sur un individu.

63. Entretien avec un capitaine instructeur à l'EOGN, Saint Astier.

64. Une autre variante, réalisée en Nouvelle Calédonie, met en mouvement la réserve qui accomplit la charge "sans haine" tandis que les assiégés demeurent en position de barrage ; "ça permet de souffler sans avoir à attaquer" (chef d'escadron déplacé en Nouvelle-Calédonie). Ce dispositif neutralisait des ressentiments exacerbés à l'encontre de femmes canaques qui exaspéraient les gendarmes parce qu'elles utilisaient des protections hygiéniques comme projectiles.

65. Impression prise sur le vif, lors d'un exercice de décrochage à Saint Astier.

66. Instruction 7001, op cit.

67. Entretien avec un Lt-colonel, instructeur à l'EOGN, Saint Astier.

68. Le service de la gendarmerie, $\mathrm{N}^{\circ} 36$, missions de maintien de l'ordre, tome III, l'équipe légère d'intervention, centre de documentation de la direction de la gendarmerie. Document d'une centaine de pages.

69. J'emprunte les notions de "violence colérique" et de "violence instrumentale" à Philippe Braud, Le jardin des délices démocratiques, FNSP, 1991, p140 et ss. 


\section{RÉSUMÉS}

Les sociétés démocratiques ont pour caractéristique essentielle de s'ouvrir au conflit, de tolérer l'existence d'un espace de désordre. Ce qui soulève deux problèmes : qu'advient-il du fameux « monopole de le violence physique légitime »? Et comment un tel Etat, avec ses moyens classiques, peut-il être capable de gérer une violence sociale elle aussi reconnue ? L'analyse du centre de perfectionnement de la gendarmerie mobile révèle la tentative de dépassement de cette contradiction : antinomie entre la volonté de façonner des agents capables de défendre le pouvoir politique jusqu'au sacrifice de leur vie et la recherche d'un répertoire sans cesse plus adapté à contrer "un citoyen momentanément égaré ». Les instructeurs de la gendarmerie prescrivent ainsi une pédagogie de la force - ce qu'on appelle la violence requise - visant à constituer un soldat de l'intérieur qui personnalisera le principe de conservation étatique. Ils imposent conjointement une pédagogie de la retenue - qu'on désigne par l'expression de violence contenue - centrée sur une stratégie de modération optimale à l'égard des " agresseurs ». La répression s'est ainsi transformée en un « maintien de l'ordre » qui vise à ordonner le désordre admissible dans le double but de ne pas produire un désordre supérieur à celui qui est enclenché par les contestataires, et de réguler, ou mieux de régulariser, une lutte ainsi fonctionnalisée.

\section{INDEX}

Mots-clés : contrôle des foules, Gendarmeries et maintien de l'ordre, Militaires, police, villes/ sociologie urbaine

Index géographique : France

Thèmes : gendarmerie nationale 